Proceedings

\title{
Novel TNBC-Targeted DOX-Arsonoliposomes ${ }^{\dagger}$
}

\author{
Maria Mantzari 1,2, Foteini Gartziou 1,2, Eleni Lambrou ${ }^{1,2}$, Spyridon Mourtas ${ }^{1,2}$, Paraskevi Zagana ${ }^{2}$ and \\ Sophia G. Antimisiaris 1,2,* \\ 1 Lab. Pharm. Technology, Department of Pharmacy, University of Patras, 26504 Rio-Patras, Greece; \\ maria.mantzari1@gmail.com (M.M.); fotini_gartz@yahoo.gr (F.G.); eleni_lamprou@upatras.gr (E.L.); \\ s.mourtas@upatras.gr (S.M.) \\ 2 Foundation for Research and Technology Hellas, Institute of Chemical Engineering Sciences, \\ FORTH/ICEHT, 26504 Rio-Patras, Greece; voulazagana@yahoo.gr \\ * Correspondence: santimis@upatras.gr; Tel.: +30-2610-962332 \\ + Presented at the 1st International Electronic Conference on Pharmaceutics, 1-15 December 2020; \\ Available online: https://iecp2020.sciforum.net/.
}

Citation: Mantzari, M.; Gartziou, F.; Lambrou, E.; Mourtas, S.; Zagana, P.; Antimisiaris, S.G. Novel TNBC-Targeted DOX-Arsonoliposomes. Proceedings 2021, 78, 17. https:// doi.org/10.3390/IECP2020-08662

Published: 1 December 2020

Publisher's Note: MDPI stays neutral with regard to jurisdictional claims in published maps and institutional affiliations.

Copyright: $(2020$ by the authors. Licensee MDPI, Basel, Switzerland. This article is an open access article distributed under the terms and conditions of the Creative Commons Attribution (CC BY) license (http://creativecommons.org/licenses/by/4.0/).

\begin{abstract}
Arsonoliposomes (ARSL) constitute a particular class of liposomes that incorporate arsonolipids (ARS) into their membranes. ARSL realize selective toxicity to cancer cells; thus, they are an important tool in the treatment of cancer. Folic acid (FA) is widely used in targeted drug delivery due to its high affinity for the folate receptors that are overexpressed in cancer cell membranes. The aim of our studies was to develop novel triple-negative breast cancer (TNBC)-targeted ARSL by incorporating folic acid-conjugated polyethylene-glycol PEG-lipid (FA-PEG-lipid) into their membrane and loading them with anticancer drug doxorubicin (DOX). ARSL incorporating $0.1 \mathrm{~mol} \%$ of FA-PEG-lipid were prepared and loaded with DOX, using the active loading protocol. They were characterized for their size distribution, zeta potential and drug entrapment efficiency (\%). Their cytotoxic activity towards TNBC cell lines, particularly MDA-MB-231 (epithelial human breast cancer cells) and MCF7 (human breast cancer cells), was evaluated by the 3-(4,5-dimethylthiazol-2-yl)2,5-diphenyltetrazolium bromide MTT-assay. The first results demonstrated enhanced toxicity of this novel type of ARSL towards cancer cells, which is particularly interesting and deserves further exploitation.
\end{abstract}

Keywords: arsonoliposomes; folic acid; doxorubicin; breast cancer; TNBC

\section{Introduction}

Arsonoliposomes (ARSL) are As(V)-containing vesicles that are composed of phospholipids, arsonolipids and cholesterol. Arsonolipids are analogues of phospholipids in which phosphorus has been replaced by arsenic in the lipid head group. It has been confirmed through in vivo and in vitro studies that specific toxicity towards cancer is shown, and that ARSL are more toxic towards cancer cells compared to normal cells. Recently, the loading of DOX into ARSL revealed that interesting synergistc effects may be demonstrated [1].

Folic acid receptor (FR)- $\alpha$ is a glycosylphosphatidylinositol-anchored membrane protein that is overexpressed in many human cancer cells, while it shows limited expression in normal cells [2]. Folic acid (FA) is used in drug delivery systems, as a tumor-targeting ligand, causing receptor-mediated endocytosis through binding of the folate conjugates to the folate receptor (FR) with high affinity, enabling nanoparticles to accumulate into cancer cells [3].

Thus, based on all mentioned above we attempted to develop novel DOX-loaded ARSL that incorporate folic acid-conjugated PEG-lipid in their membrane in order to target the triple negative cancer cells (TNBC) [4]. 


\section{Experiments}

\subsection{Materials}

1,2-Distearoyl-sn-glycerol-3-phosphatidyl-choline [DSPC] and 1,2-Distearoyl-sn-glycerol-3-phosphatidyl-ethanolamine-N-[methoxy(polyethylene-glycol)-2000] [PEG2000] were purchased from Lipoid, Germany. Cholesterol (Chol) was purchased from SigmaAldrich (Darmstadt, Germany). The rac-2,3-dipalmitoyl-oxypropylarsonic acid [ARS] (C16), was synthesized as described [1,5]. 1,2-distearoyl-sn-glycero-3-phosphoethanolamine-N-[folate(polyethylene glycol)-2000] [DSPE-PEG2000-FA] was synthesized in our laboratory. Doxorubicin hydrochloric salt (DOX) was purchased by Tocris Bioscience, UK.

For the preparation of liposomes, a bath sonicator (Branson) and a microtip-probe sonicator (Sonics and Materials, Leicestershire, UK) were used.

All other reagents and solvents used throughout the study were of analytical grade and were purchased from Sigma-Aldrich. All media used for cell growth and handling were purchased from Biochrom (Berlin, Germany).

\subsection{Methods}

\subsubsection{Preparation of Liposomes and Loading of DOX}

Liposomes of the following compositions were used in this study: (a) folate-PEGarsonoliposomes composed of DSPC/ARS/Chol/DSPE-PEG2000-MeO/folate-PEG-DSPE (12:8:10 mol/mol containing 8 mole\% PEG2000 and $0.1 \mathrm{~mole} \%$ folate-PEG-DSPE) and (b) control (non-targeted) ARSL composed of DSPC/ARS/Chol/DSPE-PEG2000-MeO (12:8:10 $\mathrm{mol} / \mathrm{mol}$ containing $8 \mathrm{~mole} \%$ PEG2000). Both liposomes were prepared by the thin-filmhydration method followed by probe sonication for size reduction. [1] The thin film is hydrated with $1 \mathrm{~mL}$ of ammonium sulfate solution $(120 \mathrm{mM}, \mathrm{pH} 5.5,300 \mathrm{mOsm})$ at $60^{\circ} \mathrm{C}$, and the ARSL suspension was sonicated with probe sonicator (Sonics and Materials, Leicestershire, UK) for approximately $10 \mathrm{~min}$ until the dispersion became completely clear. ARSL were purified from non-encapsulated ammonium sulfate by repeated ultracentrifugations for $1 \mathrm{~h}$ (each) at 60,000 rpm (Sorvall WX90 Ultra, Thermo Scientific, Waltham, MA, USA), and resuspended in PBS pH 7.4, for exchange of the dispersion media. At a lipid concentration of $1.4 \mathrm{mg} / \mathrm{mL}$ (in PBS), they were then incubated with $0.2 \mathrm{mg} / \mathrm{mL}$ DOX solution (in PBS) (corresponding to a lipid/DOX ratio equal to 7:1 (w/w)) for $60 \mathrm{~min}$ at $60^{\circ} \mathrm{C}$ for acive loading of DOX $[1,6]$. Afterwards, the ARSL were purified from the nonencapsulated drug by two repeated ultracentrifugation steps $(60,000 \mathrm{rpm}$ for $1 \mathrm{~h}$, each) and both the supernatants containing non-encapsulated DOX. The lipid content of the samples was routinely determined using a colorimetric technique (the Stewart assay), which is widely applied for phospholipids [7].

\subsubsection{Physicochemical Properties of Liposomes}

The ARSL prepared were characterized by measuring their size distribution, mean diameter and zeta-potential by DLS (dynamic light spectroscopy) with a Malvern Zetasizer 5000 (Malvern, UK). For measurement of their size, the ARSL dispersions were diluted with PBS pH 7.4 to a final concentration of $0.4 \mathrm{mg} / \mathrm{mL}$. The electrophoretic mobility of the ARSL dispersions was measured at $25^{\circ} \mathrm{C}$ by the same instrument, and zeta potential of the dispersions were calculated by application of the Helmholtz-Smolowkovski equation.

To estimate the drug concentration, DOX calibration curves in the concentration range between 5 and $40 \mu \mathrm{g} / \mathrm{mL}$ were prepared, and the final $\mathrm{D} / \mathrm{L}$ ratio $(w / w)$ was estimated and compared with the initial one for the calculation of the DOX encapsulation efficiency (\%) of each liposome type, according to Equation (1):

$$
\mathrm{EE}(\%)=\frac{\boldsymbol{D} / \boldsymbol{L}_{\text {final }}}{\boldsymbol{D} / \boldsymbol{L}_{\text {initial }}} 100
$$




\subsubsection{Cell Culture Studies}

Two types of cells were used in this study: (i) MDA-MB-231 (epithelial human breast cancer cells) and (ii) MCF7 (Human breast cancer cells). The cell lines were provided by Prof. C. Dimas, University of Thessaly, Greece. The cancer cells were grown in RPMI 1640 medium supplemented with $10 \%$ FBS and $1 \%$ antibiotic-antimycotic solution (Invitrogen, Carlsbad, CA, USA). The cells were cultured at $37^{\circ} \mathrm{C}, 5 \% \mathrm{CO}_{2} /$ saturated humidity. Medium was changed every 2-3 days.

The toxicity of the various types of ARSL towards cancer cells was evaluated by the corresponding reduction of cell viability after $24 \mathrm{~h}$ incubation with ARSL or PBS by the MTT assay [8]. For each experiment, cells were seeded overnight at $37^{\circ} \mathrm{C}$ at a density of 5 $\times 10^{4}$ cells per well, in 24 -well plates and then incubated for $24 \mathrm{~h}$ at $37^{\circ} \mathrm{C}\left(5 \% \mathrm{CO}_{2} /\right.$ saturated humidity) with $0.4 \mathrm{~mL}$ growth medium and $0.1 \mathrm{~mL}$ of the formulation evaluated in each case. Viability (\%) was calculated by the Equation (2):

$$
\text { Viability }(\%)=\frac{\mathrm{OD}-570_{\text {sample }}-\mathrm{OD}-570_{\text {background }}}{\mathrm{OD}-57 \mathrm{c}_{\text {control }}-\mathrm{OD}-570_{\text {background }}} \times 100
$$

where OD-570 control corresponds to untreated cells (or PBS control) and OD-570 background to MTT without cells. All samples were diluted in order to achieve DOX concentrations of 1 $\mu \mathrm{M}$ and $3 \mu \mathrm{M}$.

For the evaluation of DOX uptake of ARSL (targeted or not) by cultured MDA-MB231 and MCF7 cells, cells were seeded overnight at density of $12 \times 10^{4}$ cells $/ \mathrm{mL}$ per well in 12-well plates in RPMI at $37{ }^{\circ} \mathrm{C}\left(5 \% \mathrm{CO}_{2} /\right.$ saturated humidity) and then incubated with DOX liposomes for $1 \mathrm{~h}$. DOX concentration was $10 \mu \mathrm{M}$ (non toxic for $1 \mathrm{~h}$ incubation). After treatment, the cells were washed twice with PBS and then detached and lysed by the addition of $0.9 \mathrm{~mL}$ of PBS and $0.1 \mathrm{~mL}$ of Triton X-100 (20\%). The samples were collected and their fluorescence intensity was measured (EX 490 and EM $520 \mathrm{~nm}$ ). The protein concentration in every sample was measured by the Bradford microassay. Finally, DOX uptake by cells was expressed as \% uptake/protein (by comparison of the DOX measured in the cells and the total amount of DOX incubated with the cells).

\section{Results}

\subsection{Physicochemical Properties of Liposomes}

All liposome dispersions were characterized for their size distribution (mean hydrodynamic diameter and polydispersity index) and their zeta-potential, and the results are presented in Table 1.

Table 1. Physicochemical properties of arsonoliposomes (ARSL) and folic acid (FA)-ARSLbefore and after doxorubicin (DOX) loading, formulated in PBS buffer ( $\mathrm{pH} 7.40)$.

\begin{tabular}{|c|c|c|c|c|}
\hline Formulations & $\begin{array}{l}\text { Mean Hydrodynamic } \\
\text { Diameter (nm) }\end{array}$ & PI & $\zeta$-Potential (mV) & DOX Loading (\%) \\
\hline ARSL & $118.9 \pm 0.8$ & 0.231 & $-6.73 \pm 0.29$ & \\
\hline DOX-ARSL & $143.1 \pm 1.8$ & 0.421 & $-6.35 \pm 0.59$ & $94.9 \pm 1.8$ \\
\hline FA-ARSL & $113.6 \pm 1.4$ & 0.265 & $-7.32 \pm 0.64$ & - \\
\hline DOX-FA-ARSL & $126.9 \pm 0.6$ & 0.410 & $-8.78 \pm 0.54$ & $95.9 \pm 2.4$ \\
\hline
\end{tabular}

The mean hydrodynamic diameter of all ARSL was in the nano-range, between 113 and $143 \mathrm{~nm}$. The polydispersity index values were between 0.231 and 0.421 for all ARSL: the lowest for ARSL and the highest for DOX- FA-ARSL. All samples were monodisperse as observed in DLS reports (non-shown). The addition of FA-lipid doesn't seem to alter the vesicle mean diameter and the PDI. However, DOX-ARSL appear to have increased mean diameter and PDI, compared to all other formulations. Results of zeta potential measurements were in the range between -6.35 and $-8.78 \mathrm{mV}$, which are anticipated (low negative values) due to pegylation. The loading of DOX was achieved in all ARSL types, 
and loading EE\% ranged between $95 \%$ and $96 \%$ when the incubation of DOX with empty vesicles was carried out at $60^{\circ} \mathrm{C}$. There is no significant difference in DOX-loading values between arsonoliposmes and FA-arsonoliposomes (Table 1), suggesting that the addition of FA-lipid does not affect the loading capacity of the vesicle.

\subsection{In Vitro Toxicity}

The cytotoxicity of DOX-ARSL and DOX-FA-ARSL was carried out with two cancer cell lines, MDA-MB-231 and MCF7 cells. The appropriate D/L ratios were used for preparation of the DOX-loaded liposomes, specifically a $0.05 \mathrm{~mole} / \mathrm{mole}$ ratio in order to have $1 \mu \mathrm{M}$ of DOX. As seen on Figure 1A, a higher anticancer effect of DOX-FA-ARSL is evident towards MDA-MB-231, compared to all other formulatons: a result consistent with the fact that these cells are known to have high FR expression. On the contrary, DOX-FAARSL anticancer activity towards MCF7 cells was not significantly different than that of the other formulations (non-targeted), which is logical since these cells do not overexpress the FR receptor (Figure 1B).

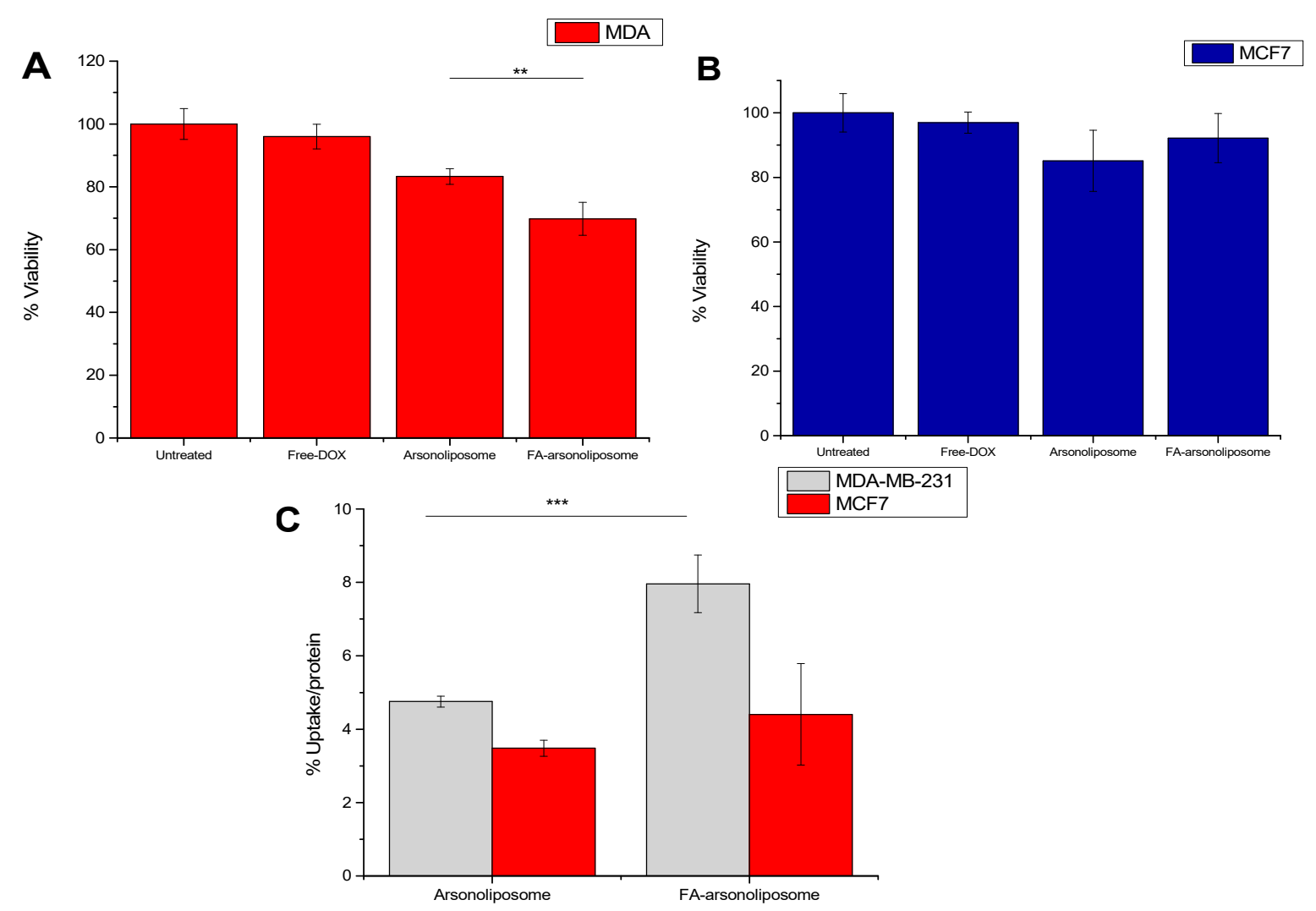

Figure 1. Viability of cells after incubation with DOX-ARSL, DOX-FA-ARSL and Free-DOX $1 \mu M$ for 24 h. (A) MDA-MB231 (epithelial human breast cancer cells). (B) MCF7. (C) Uptake of DOX by MDA-MB-231 and MCF7 cells after $1 \mathrm{~h}$ of coincubation of cells with DOX- ARSL (targeted and non-targeted). ${ }^{* *}$ corresponds to $p<0.01 .{ }^{* *}$ corresponds to $p<0.001$.

The previous results are verified also by the DOX uptake results for the two cell types, which are in good agreement with the viability results. Indeed, as seen in Figure 1C, the uptake of DOX by MDA-MB-231 cells is substantially increased when DOX-FA-ARSL are used compared to non-targeted ARSL, while a similar effect was not observed in the case of the MCF7 cells.

\section{Discussion}

Herein, we investigated the potential of novel TNBC-targeted ARSL by incorporating folic acid-conjugated PEG-lipid in their membrane and loading them with the anticancer drug DOX. Both ARSL and FA-ARSL had mean diameters in the nano-range, between 120 
and $143 \mathrm{~nm}$ (Table 1 ), polydispersity values below $<0.4$ and slightly negative zeta potential values and could be efficiently loaded with DOX by an active-loading method.

The in vitro experiments on the cancer cells MDA-MB-231 and MCF7, two of the most commonly studied TNBC cell lines, presented hopefull results. The uptake of DOX by MDA-MB-231 cells is significantly higher from targeted ARSL (with 0.1\%FA) compared with non-targeted ones, suggesting that ARSL can be targeted using folic acid as a ligand. Moreover, the cytotoxic effect of DOX-FA-ARSL towards cancer cells was higher than that of free DOX and DOX-ARSL, indicating the importance of combining both compounds in the same vesicular structure.

\title{
5. Conclusions
}

The current results indicate the potential antitumor properties of the novel folic acid targeted ARSL that incorporate doxorubicin towards triple-negative breast cancer (TNBC), which merits further study in the future.

\begin{abstract}
Author Contributions: S.G.A. conceived the experiments and got the funding. S.M. designed the synthesis of DSPE-PEG-FE lipid and the molar composition of each liposome. F.G. designed and helped with the execution of the cell experiments. P.Z. conceived the experiments regarding the cells and the loading of drug into arsonoliposomes. M.M. performed all the experiments needed together with E.L. M.M. wrote a first draft of the paper. M.M., F.G. and E.L. gathered and analyzed the data. S.A. corrected the draft paper and wrote the final version. All authors have read and agreed to the published version of the manuscript.
\end{abstract}

Data Availability Statement: The data presented in this study are available on request from the corresponding author.

Funding: This research has been co-financed by the European Regional Development Fund of the European Union and Greek national funds through the Operational Program Competitiveness, Entrepreneurship and Innovation, under the call RESEARCH-CREATE-INNOVATE (project code: MIS5031802).

Conflicts of Interest: The authors declare no conflict of interest. The funders had no role in the design of the study; in the collection, analyses, or interpretation of data; in the writing of the manuscript, and in the decision to publish the results.

\section{References}

1. Zagana, P.; Mourtas, S.; Basta, A.; Antimisiaris, S.G. Preparation, Physicochemical Properties, and In Vitro Toxicity towards Cancer Cells of Novel Types of Arsonoliposomes. Pharmaceutics 2020, 12, 327, doi:10.3390/pharmaceutics12040327.

2. Choi, Y.W.; Kang; Park; Kang, M.J.; Park, S.H.; Kang, M.H.; Park, M.J. Folic acid-tethered Pep-1 peptide-conjugated liposomal nanocarrier for enhanced intracellular drug delivery to cancer cells: Conformational characterization and in vitro cellular uptake evaluation. Int. J. Nanomed. 2013, 8, 1155-1165, doi:10.2147/ijn.s39491.

3. Ye, P.; Zhang, W.; Yang, T.; Lu, Y.; Lu, M.; Gai, Y.; Ma, X.; Xiang, G. Folate receptor-targeted liposomes enhanced the antitumor potency of imatinib through the combination of active targeting and molecular targeting. Int. J. Nanomed. 2014, 9, 2167-2178, doi:10.2147/ijn.s60178.

4. Gabizon, A.A.; Patil, Y.; La-Beck, N.M. New insights and evolving role of pegylated liposomal doxorubicin in cancer therapy. Drug Resist. Updat. 2016, 29, 90-106, doi:10.1016/j.drup.2016.10.003.

5. Ioannou, P.V.; Tsivgoulis, G.M. A high yield procedure for the preparation of arsonolipids (2,3-diacyloxypropylarsonic acids). Chem. Phys. Lipids 2010, 163, 51-55, doi:10.1016/j.chemphyslip.2009.10.008.

6. Zucker, D.; Marcus, D.; Barenholz, Y.; Goldblum, A. Liposome drugs' loading efficiency: A working model based on loading conditions and drug's physicochemical properties. J. Control. Release 2009, 139, 73-80.

7. Stewart, J.C.M. Colorimetric determination of phospholipids with ammonium ferrothiocyanate. Anal. Biochem. 1980, 104, 10-14.

8. Morgan, D.M.L. Tetrazolium (MTT) Assay for Cellular Viability and Activity. In Polyamine Protocols. Methods in Molecular Biology ${ }^{\mathrm{TM}}$; Morgan, D.M.L., Ed.; Humana Press: Totowa, NJ, USA, 1998; Volume 79. 